\title{
Lipoic Acid Decreases the Expression of Poly ADP-Ribose Polymerase and Inhibits Apoptosis in Diabetic Rats
}

This article was published in the following Dove Press journal: Diabetes, Metabolic Syndrome and Obesity: Targets and Therapy

\author{
Jingfang Chen' \\ Qiang $\mathrm{Li}^{2}$ \\ 'Department of Endocrinology, Henan \\ Provincial Chest Hospital, Zhengzhou, \\ Henan Province, People's Republic of \\ China; ${ }^{2}$ Department of Endocrinology, \\ Shenzhen University General Hospital, \\ Shenzhen, Guangdong Province, People's \\ Republic of China
}

Purpose: To study the effects of lipoic acid on poly ADP-ribose polymerase (PARP) expression and apoptosis in diabetic rats.

Materials and Methods: Sprague-Dawley rats $(n=30)$ with high-fat diet- and streptozotocin-induced diabetes were randomly divided into two groups: diabetic model (DM) group and lipoic acid (LA) treatment group; another 10 rats were selected as normal controls (NC). The serum levels of 8-hydroxy-2'-deoxyguanosine, nitrotyrosine, and 8-isoprostane; sciatic nerve cell apoptosis index; and PARP expression were detected in the rats, and morphological changes in the sciatic nerve were recorded.

Results: The blood glucose level in the DM and LA groups was significantly higher than that of the NC group $(\mathrm{P}<0.01)$. Compared to the NC group, the DM group showed demyelinating changes to sciatic nerve fibers. PARP expression; serum levels of 8-hydroxy-2-deoxyguanosine, nitrotyrosine, and 8-isoprostane; and the apoptosis index of sciatic nerve cells were significantly higher than those of the NC group $(\mathrm{P}<0.01)$. Following LA treatment, the above indices showed significant improvement $(\mathrm{P}<0.01)$.

Conclusion: Lipoic acid may improve the symptoms of diabetic neuropathy by reducing PARP activity and inhibiting apoptosis.

Keywords: lipoic acid, diabetic peripheral neuropathy, poly ADP-ribose polymerase, oxidative stress, apoptosis

\section{Introduction}

The prevalence of diabetic neuropathy in diabetic patients is very high. It is one of the most common chronic complications of diabetes. The damage can affect the central and peripheral nerves, and the incidence of diabetic peripheral neuropathy is high, which is the current leading cause of disability and death in people with diabetes. ${ }^{1}$ The pathogenesis of diabetic peripheral neuropathy is complex and hence not yet fully understood. Oxidative stress theory is understood to be one of the main mechanisms of pathogenesis. Excessive free radicals cause DNA damage resulting in cellular apoptosis, which in turn initiates a series of diabetic complications including peripheral neuropathy. ${ }^{2}$ Poly ADP-ribose polymerase (PARP) is a multifunctional enzyme mainly involved in the repair of DNA and plays an important role in the initiation of apoptosis. ${ }^{3,4}$ PARP can catalyze ADP ribose from NAD+ and transfer it one by one to the next substrate molecule such as histone, laminin, DNA polymerase, topoisomerase, or PARP itself. This process allows PARP to play a role in repairing DNA damage,
Correspondence: Qiang Li

Department of Endocrinology, Shenzhen University General Hospital, Shenzhen

City, Guangdong Province 518055,

People's Republic of China

Email 332706770@qq.com 
maintaining the stability of the genome, affecting apoptosis, etc., which is very significant in the occurrence and development of diabetes and its chronic complications. ${ }^{5}$

Currently, lipoic acid (LA) is the strongest natural antioxidant known, with both fat- and water-soluble properties. ${ }^{6-8}$ It can remove active oxygen free radicals in the body and inhibit many redox-sensitive signaling pathways. In addition, LA is also a key auxiliary group of various enzymes such as pyruvate dehydrogenase and $\alpha$-ketoglutarate dehydrogenase in the mitochondria, which can promote and improve oxidative phosphorylation and stabilize the mitochondrial respiratory chain, improve mitochondrial function, and reduce the occurrence of cellular apoptosis or death. ${ }^{9}$ Some studies have shown that LA can also increase blood flow to nerve vessels, improve nerve conduction speed, and increase $\mathrm{Na}+, \mathrm{K}+$-ATPase activities, all of which can significantly improve the symptoms of diabetic neuropathy. ${ }^{10}$ In this study, we observed changes in PARP expression and cellular apoptosis after LA treatment in a rat model of diabetes, and further explored the protective mechanism of LA on diabetic peripheral neuropathy.

\section{Materials and Methods}

\section{Ethics Statement}

This study was carried out in strict accordance with the recommendations in the Guide for the Care and Use of Laboratory Animals of the National Institutes of Health. All protocol were performed in accordance with the approved guidelines, which were approved by the Ethics Committee of Harbin Medical University.

\section{Materials}

Eight-week-old, male Sprague-Dawley rats $(\mathrm{n}=40$; body weight, 210-240 g) (Beijing Weitonglihua Experimental Animal Technology Co. Ltd., Beijing, China); streptozotocin (STZ) and LA (Sigma company, Saint Louis, USA); 8-hydroxy-2-deoxyguanosine enzyme-linked immunosorbent assay (ELISA) kit and nitrotyrosine ELISA kit (Shanghai Bio-Swamp Life Science, Shanghai, China); 8-isoprostane ELISA kit (Bioswamp Life Science Lab, Wuhan, China); terminal deoxynucleotidyl transferase dUTP nick end labeling (TUNEL) apoptosis in situ detection kit (F. Hoffmann-La Roche Ltd., Basel, Switzerland); and PARP antibody (Beijing Biosynthesis Biotechnology Co., Ltd., Beijing, China).

\section{Methods}

\section{Diabetic Rat Model and Grouping}

In all, male Sprague Dawley rats were adaptively fed for one week. Ten randomly selected rats were assigned to the normal control (NC) group and were given ordinary feed. The remaining 30 rats were fed a high-fat diet for four weeks. The 30 rats were fasted for $12 \mathrm{~h}$, following which a low-dose STZ (35 mg/kg) solution (dissolved STZ powder with $0.1 \mathrm{~mol} / \mathrm{L}$ sodium citrate buffer solution, $\mathrm{pH}$ 4.4) was intraperitoneally injected. Rats in the $\mathrm{NC}$ group were injected with only the $0.1 \mathrm{~mol} / \mathrm{L}$ citrate buffer after $12 \mathrm{~h}$ of fasting. Blood glucose was measured from the tail tip $72 \mathrm{~h}$ later, by using a blood glucose meter (Roche, Germany). Blood glucose levels $\geq 16.7 \mathrm{mmol} / \mathrm{L}$ indicated model success. During this process, two rats whose blood glucose levels were less than $16.7 \mathrm{mmol} / \mathrm{L}$ were excluded, and two rats died. Thus, the final analysis was conducted on 26 diabetic rats that were randomly divided into two groups ( $\mathrm{n}=13$ each) - the diabetic model (DM) group and LA treatment group. One week later, the LA group was intraperitoneally injected with LA $(100 \mathrm{mg} / \mathrm{kg})$, while the DM and NC groups were injected with the corresponding volume of normal saline, once a day for 8 weeks. Body weight and blood glucose were measured every 2 weeks.

\section{Specimen Collection}

Eight weeks later, 10 rats in each group were randomly selected, weighed, and anesthetized with $2 \%$ pentobarbital sodium solution. A blood sample was collected via femoral puncture and centrifuged at $3000 \mathrm{rpm}$ for 10 min; the supernatant was stored at $-20^{\circ} \mathrm{C}$ until further analysis. The sciatic nerve was fixed using $4 \%$ polyformaldehyde, and paraffin sections were prepared according to standard protocol.

\section{Detection of Serum Indicators}

The serum levels of 8-hydroxy-2-deoxyguanosine, nitrotyrosine, and 8-isoprostane were detected by ELISA kits, according to the manufacturers' instructions.

\section{Morphological Observation of the Sciatic Nerve}

After the sciatic-nerve paraffin sections were stained with hematoxylin-eosin (HE) and for myelin staining (solochrome cyanine $\mathrm{R}$ method), the pathological morphology of the sciatic nerves was observed under light microscopy and photographed.

The myelin staining process is as follows: 
(a) Fix the removed tissue in $20 \%$ formaldehyde for more than 3 days, and embed it in paraffin after dehydration to make 4-6 mm-thick paraffin sections.

(b) After the section is dewaxed, add solochrome cyanine $\mathrm{R}$ solution to the section, stain at room temperature for $20 \mathrm{~min}$, drain the staining solution, and rinse with running water for $2 \mathrm{~min}$.

(c) Continue to soak in a $10 \%$ aqueous solution of ferric ammonium sulfate for about $3 \mathrm{~min}$, until the collagen fibers and muscle fibers are almost colorless or light gray and the nerve myelin sheath is a clear blue.

(d) Then, rinse with water for $10 \mathrm{~min}$, counter-stain with the fluorescent pink dye solution for $10 \mathrm{~s}$, rinse again with water, then dehydrate with alcohol, seal with xylene and neutral resin, and observe and photograph under a light microscope.

\section{Detection of Apoptosis of Sciatic Nerve Cells}

The sciatic-nerve paraffin sections were stained according to the manufacturer's instructions for the TUNEL kit. Microscopic brown particles seen in the nucleus were considered as positive staining. We randomly selected five consecutive, non-repetitive fields from each section, under a high-power microscope. The numbers of positive cells and the total numbers of nerve cells were counted, which were used to calculate the apoptotic index of sciatic nerve cells. Apoptosis index (AI)=positive cells/total number of nerve cells.

\section{Detection of PARP Expression in the Sciatic Nerve}

Brown-yellow particles present in the cytoplasm indicated a positive expression. We randomly selected five consecutive, non-repetitive fields from the same tissue section under high magnification $(\times 400)$. The average optical density (OD) was measured by Image-Pro Plus 6.0 software (Media Cybernetics Inc., Maryland, USA), which expressed the relative concentration of positive PARP expression.

\section{Statistical Analysis}

SPSS 17.0 software (SPSS Inc., Chicago, USA) was used for all statistical analyses. The mean \pm standard deviation $(\overline{\mathrm{X}} \pm \mathrm{s})$ was used to represent the measurement data, and one-way analysis of variance was used to compare the means of each group. $\mathrm{P}<0.05$ indicated a statistically significant difference.

\section{Results}

\section{Establishment of a Diabetic Rat Model}

In the process of establishing a DM, two rats died, two rats did not develop diabetes, and one rat died in the LA group. The serum and tissue samples were analyzed in the three groups ( $\mathrm{n}=10$ /group). As shown in Table 1, the blood glucose levels in the DM and LA groups were significantly higher than in the $\mathrm{NC}$ group $(\mathrm{P}<0.01)$. This showed that the DM was successfully established. Further, compared with before LA treatment, there was no statistical difference in blood glucose after treatment $(\mathrm{P}>0.05)$; hence, it could be said that LA had no significant effect on blood glucose in diabetic neuropathy.

\section{Morphological Observation of the Sciatic Nerve}

HE staining: Microscopically, the myelinated nerve fibers of the sciatic nerve in the NC group were normal and closely arranged, the axon and its surrounding myelin sheath structures were clear, and the stroma was evenly stained (Figure 1A). However, in the DM group, the arrangement of nerve fibers was disordered, the nerve fibers were demyelinated, and the axons had atrophied or even disappeared (Figure 1B). After LA treatment, the pathological changes noted to the sciatic nerve were significantly alleviated, with only some nerve fibers showing slight demyelination and significantly lesser axonal atrophy than that in the DM group (Figure 1C).

Myelin staining: In the NC group, the myelin sheath structure was normal and intact (Figure 2A). There were significant changes in demyelination of nerve fibers in the DM group (Figure 2B). Following LA treatment, the demyelinated lesions of nerve fibers were gradually improved, and the sciatic nerve specimens were only slightly demyelinated as compared to that in the DM group (Figure 2C).

Table I Blood Glucose Levels of Rats Before and After Treatment in Three Groups

\begin{tabular}{|l|l|l|}
\hline $\begin{array}{l}\text { Group } \\
(\mathbf{n}=1 \mathbf{0})\end{array}$ & $\begin{array}{l}\text { BG After Diabetic } \\
\text { Modeling (mmol/L) }\end{array}$ & $\begin{array}{l}\text { BG After LA } \\
\text { Treatment (mmol/L) }\end{array}$ \\
\hline DM & $26.15 \pm 2.40$ & $25.94 \pm 2.17$ \\
LA & $24.94 \pm 2.47$ & $24.75 \pm 2.78$ \\
NC & $5.21 \pm 0.85$ & $5.32 \pm 1.25$ \\
\hline
\end{tabular}

Abbreviations: BG, blood glucose; DM, diabetic model group; LA, lipoic acid treatment group; NC, normal control group. 

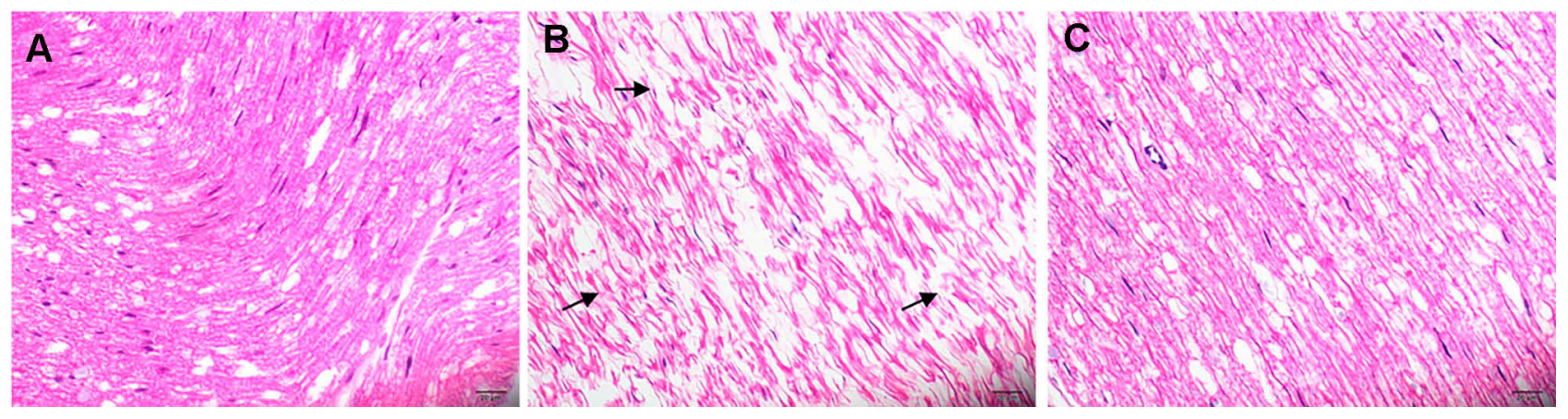

Figure I Histological analysis of sciatic nerve tissue (hematoxylin-eosin staining, $\times 400$ ). Myelinated nerve fibers of the sciatic nerve in the NC group appear normal and are closely arranged, the axon and its surrounding myelin sheath structure are clear, and the stroma is evenly stained (A). As indicated by the arrow, the arrangement of nerve fibers is disordered, the nerve fibers are demyelinated, with atrophied or even absent axons disappeared in the DM group (B). After LA treatment, the pathological changes in the sciatic nerve show significant improvement (C).

Abbreviations: DM, diabetic model; LA, lipoic acid treatment; NC, normal control.
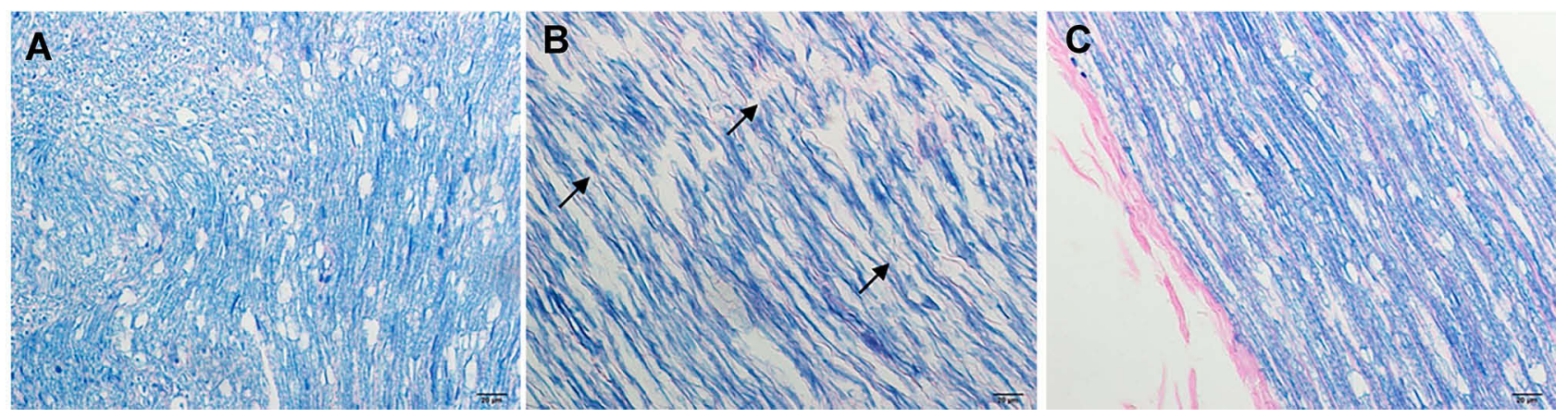

Figure 2 Myelin staining (Solochrome cyanine R method) $(\times 400)$. The myelin sheath structure was normal and intact in the NC group $(\mathbf{A})$ Significant changes can be seen in demyelination of nerve fibers in the DM group (arrows, B). Following LA treatment, the sciatic nerve showed only mild demyelination as compared to the DM group (C). Abbreviations: DM, diabetic model; LA, lipoic acid treatment; NC, normal control.

\section{Effect of LA on Levels of Oxidative Stress Indices}

The three oxidative stress indices-8-hydroxy-2-deoxyguanosine, nitrotyrosine, and 8-isoprostane-in the DM group were significantly greater than those in the $\mathrm{NC}$ group $(\mathrm{P}<0.01)$. After LA treatment, the above indices markedly decreased $(\mathrm{P}<0.01)$ (Table 2$)$.

Table 2 Effect of Lipoic Acid on Levels of Oxidative Stress Indices

\begin{tabular}{|l|l|l|l|}
\hline $\begin{array}{l}\text { Group } \\
(\mathbf{n = 1 0})\end{array}$ & $\begin{array}{l}\text { 8-Hydroxy-2'- } \\
\text { Deoxyguanosine } \\
(\mathbf{p g} / \mathbf{m L})\end{array}$ & $\begin{array}{l}\text { Nitrotyrosine } \\
(\mathbf{n m o l} / \mathbf{L})\end{array}$ & $\begin{array}{l}\text { 8-Isoprostane } \\
\mathbf{( p g / m L})\end{array}$ \\
\hline DM & $127.59 \pm 3.08$ & $566.29 \pm 23.96$ & $83.09 \pm 2.96$ \\
LA & $121.77 \pm 5.27$ & $478.88 \pm 39.30$ & $64.41 \pm 2.39$ \\
NC & $114.56 \pm 7.21$ & $450.09 \pm 13.89$ & $59.30 \pm 3.05$ \\
\hline
\end{tabular}

Abbreviations: DM, diabetic model group; LA, lipoic acid treatment group; NC, normal control group.

\section{Comparison of Apoptosis of Sciatic Nerve Cells}

The apoptosis of sciatic nerve cells was detected by TUNEL staining, and the apoptosis index (AI) was calculated. The results showed that $\mathrm{AI}$ in the $\mathrm{DM}$ group was significantly higher than that in the $\mathrm{NC}$ group $(\mathrm{P}<0.01)$, but $\mathrm{AI}$ decreased significantly after LA treatment $(\mathrm{P}<0.01)$ (Table 3$)$.

Table 3 Effect of Lipoic Acid on PARP Expression and Apoptosis Index

\begin{tabular}{|l|l|l|}
\hline Group $(\mathbf{n}=\mathbf{1 0})$ & Al & OD \\
\hline DM & $0.65 \pm 0.05$ & $58.56 \pm 4.51$ \\
LA & $0.52 \pm 0.03$ & $26.44 \pm 2.64$ \\
NC & $0.36 \pm 0.03$ & $8.43 \pm 1.69$ \\
\hline
\end{tabular}

Abbreviations: PARP, poly ADP-ribose polymerase; Al, apoptosis index; OD, optical density; DM, diabetic model group; LA, lipoic acid treatment group; NC, normal control group. 


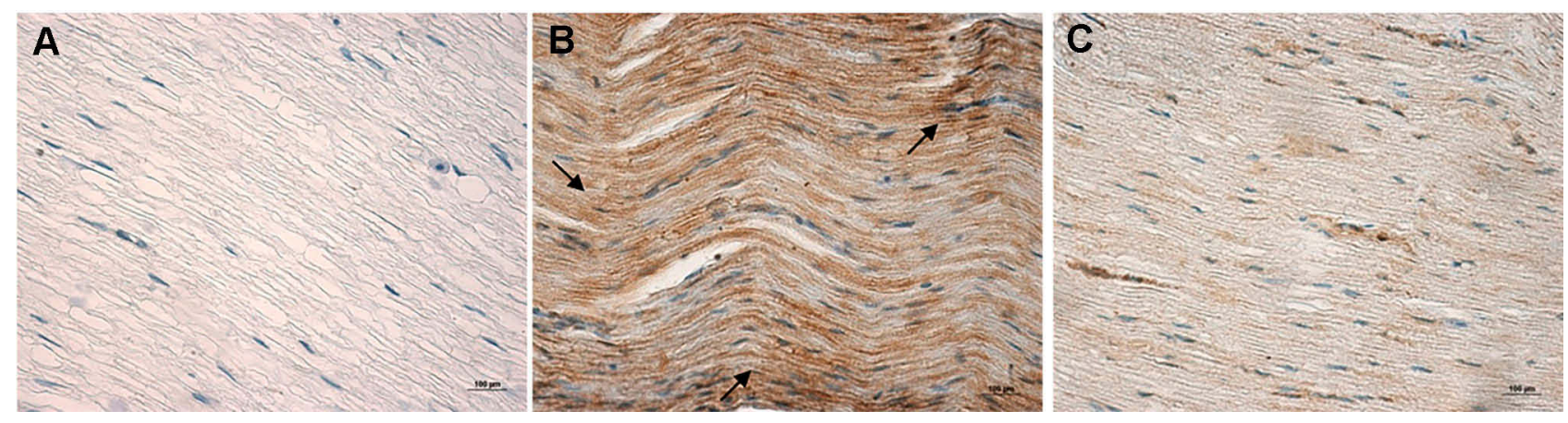

Figure 3 Immunohistochemical staining $(\times 400)$ for PARP expression of the sciatic nerve. No brown staining observed in the sciatic nerve tissue of the NC group (A). In the DM group, many brown-stained particles can be seen (B). After LA treatment, the sciatic nerve showed a light brown color, almost similar to that of the NC group (C). Abbreviations: PARP, poly ADP-ribose polymerase; DM, diabetic model; LA, lipoic acid treatment; NC, normal control.

\section{Expression of PARP in the Sciatic Nerve}

Microscopic examination showed no brown staining of the sciatic nerve in the NC group (Figure 3A), but many brown particles could be seen in the DM group (Figure 3B). After LA treatment, the sciatic nerve showed a light brown color, which was close to the color observed in the $\mathrm{NC}$ group (Figure 3C). The results of OD showed that the expression of PARP in the sciatic nerve of the DM group was significantly higher than that of the $\mathrm{NC}$ group $(\mathrm{P}<0.01)$, but was significantly lower after treatment with LA $(\mathrm{P}<0.01)$ (Table 3$)$.

\section{Discussion}

The mechanism of diabetic peripheral neuropathy is very complex ${ }^{1}$ and hence not very well understood. It may be associated with the interaction of metabolic disorders (including hyperglycemia toxicity, the aldose reductase-polyol pathway, production of glycosylation end products, oxidative stress, abnormal lipid metabolism); microvascular injury; neurotrophic factor deficiency; and abnormal release of cytokines and autoimmune factors. An increasing number of studies have shown that oxidative stress is the common mechanism of diabetic peripheral neuropathy, ${ }^{11,12}$ which has attracted the attention of researchers in recent years. The persistently high level of blood glucose in diabetic patients increases the level of oxidative stress, resulting in excessive production of reactive oxygen species (ROS), inhibition of some cytokines and cytochrome oxidase required for glucose metabolism, and, at the same time, reduction of the body's antioxidant capacity. It directly leads to denaturation of intracellular proteins and various enzymes, while the accumulation of inactive proteins in cells may reduce some nutritional factors and neurotransmitters transmitted from the synapses to cells, which in turn can lead to apoptosis.

Our results showed that compared with the NC group, the DM and LA groups showed significantly increased blood glucose levels after 4 weeks of high-fat diet combined with low-dose STZ, which was in accordance with the standard for a diabetic rat model. ${ }^{13,14} 8$-hydroxy-2'deoxyguanosine, nitrotyrosine, and 8-isoprostane are the products of tissue cell injury under oxidative stress and can be used to evaluate tissue damage. ${ }^{15,16}$ The concentrations of 8-hydroxy-2-deoxyguanosine, nitrotyrosine, and 8 -isoprostane and the sciatic nerve cell apoptosis index in the DM group were significantly higher than those in the NC group $(\mathrm{P}<0.01)$, suggesting that peripheral nerve injury in hyperglycemic conditions may be caused by oxidative stress. ${ }^{17,18}$ Excessive superoxide anions are produced in the mitochondria of cells in a state of hyperglycemia. When all types of ROS and reactive nitrogen species exceed the scavenging ability of the body, an imbalance is created between the oxidation and antioxidant systems in vivo, leading to varied types of tissue stress damage.

The initiation of oxidative stress is also accompanied by the activation of PARP. ${ }^{19}$ Hyperglycemia of diabetic neuropathy increases ROS and reactive nitrogen species in the body, causing DNA strand damage and cleavage, which in turn increases PARP activity, inhibits glyceraldehyde phosphate dehydrogenase, and prevents glucose metabolites from passing through normal aerobic oxidation. This process activates reaction pathways such as polyol pathway, glycosylation end product pathway, protein kinase $\mathrm{C}$ (PKC) pathway, and hexosamine pathway, resulting in a series of damaging factors that directly damage vascular endothelial cells and induce 
apoptosis. $^{20,21}$ PARP also plays an important role in the pathogenesis of other diseases related to oxidative stress, such as cardiovascular and tumor-related diseases, via oxidative stress damaging neurons and vascular endothelial cells. ${ }^{22,23}$ The damage to tissue cells caused by PARP under oxidative stress is the pathological basis for the occurrence and development of diabetic peripheral nerve complications. Therefore, improving the state of oxidative stress and inhibiting the activity of PARP is one of the most effective measures for prevention and treatment of diabetic peripheral neuropathy.

Lipoic acid can effectively eliminate key free radicals such as ROS, superoxide anion, and peroxynitrite that can cause death of vascular endothelial cells and nerve cells under oxidative stress. ${ }^{24}$ Furthermore, it can effectively alleviate or even eliminate the symptoms of diabetic peripheral neuropathy by improving blood flow of neurotrophic vessels, nutritional status and conduction velocity of peripheral nerve, and function of vascular endothelial cells to ultimately improve the quality of life of patients. ${ }^{25}$

In conclusion, our results suggest that LA can likely improve the symptoms of diabetic peripheral neuropathy by inhibiting oxidative stress-induced DNA damage, decreasing the activity of PARP, and inhibiting apoptosis in nerve cells. This provides a potential theoretical basis for the clinical treatment of diabetic peripheral neuropathy with LA.

\section{Acknowledgments}

The study was supported by a grant from the Heilongjiang Science and Technology Research Program (no. GA07C303107) as part of the Capital Characteristic Clinical Application Research project.

\section{Author Contributions}

All authors contributed to data analysis, drafting and revising the article, gave final approval of the version to be published, and agree to be accountable for all aspects of the work.

\section{Disclosure}

The authors declare no competing interests.

\section{References}

1. Saikat D, Sonjit D, Kumar DA, et al. Molecular mechanism of diabetic neuropathy and its pharmacotherapeutic targets. Eur J Pharmacol, 2018;15(833):472-523
2. Brownlee M Biochemistry and molecular biology of diabetic complications. Nature, 2001, 414(6865):813-820. doi:10.1038/ 414813a

3. Makogon NV, Aleksieieva IM [Poly(ADP-ribose) polymerase (PARP): physiological and pathological roles]. Fiziol Zh, 2012, 58 (3):95-112. Ukrainian. doi:10.15407/fz58.03.095

4. Hana H, Ilona K, Demin AA, et al. The importance of poly (ADP-Ribose) polymerase as a sensor of unligated okazaki fragments during DNA replication. Mol Cell, 2018;71(2):319-331. doi:10.1016/ j.molcel.2018.06.004

5. Luo X, Kraus WL A one and a two ... expanding roles for poly (ADP-ribose) polymerases in metabolism. Cell Metab, 2011, 13 (4):0-355. doi:10.1016/j.cmet.2011.03.011

6. Chukanova EI, Chukanova AS Alpha-lipoic acid in the treatment of diabetic polyneuropathy. Zh Nevrol Psikhiatr Im S S Korsakova, 2018, 118(1):103-109. doi:10.17116/jnevro201811811103-109

7. Tasci I, Demir C, Kuloglu T Effects of alpha lipoic acid on loss of myelin sheath of sciatic nerve in experimentally induced diabetic rats. Med Arch, 2018, 72(3):178-181. doi:10.5455/medarh.2018.72.178181

8. Strokov IA, Phokina AS $\alpha$-Lipoic acid as the main pharmacological drug for in- and outpatient treatment of diabetic polyneuropathy. $Z \mathrm{~h}$ Nevrol Psikhiatr Im $S \quad S$ Korsakova, 2017, 117(3):50-55. doi:10.17116/jnevro20171173150-55

9. Coppey LJ, Gellett JS, Davidson EP, et al. Preventing superoxide formation in epineurial arterioles of the sciatic nerve from diabetic rats restores endothelium-dependent vasodilation. Free Radic Res, 2003, 37:33-40. doi:10.1080/1071576021000028442

10. Jain SK, Lim G Lipoic acid decreases lipid peroxidation and protein glycosylation and increases $(\mathrm{Na}+\mathrm{K})$ - and Ca-ATPase activities in high glucose-treated human erythrocytes. Free Radic Biol Med, 2001, 29(11):1122-1128. doi:10.1016/S0891-5849(00)00410-X

11. Coppey L, Lu B, Gerard C, et al. Effect of inhibition of angiotensin-converting enzyme and/or neutral endopeptidase on neuropathy in high-fat-fed C57Bl/6J mice. $J$ Obes, 2012, 2012:1-10. doi: $10.1155 / 2012 / 326806$

12. Singh R, Kishore L, Kaur N Diabetic peripheral neuropathy: current perspective and future directions. Pharmacol Res, 2014, 80:21-35. doi:10.1016/j.phrs.2013.12.005

13. Zhang B, Yang Y, Luo B, et al. Effects of different foods on blood glucose and lipid in type 2 diabetes mellitus in a rat model. $J$ Surg Res 2018;229:254-261. doi:10.1016/j.jss.2018.02.045

14. Zhou J, Du X, Long M, et al.Neuroprotective effect of berberine is mediated by MAPK signaling pathway in experimental diabetic neuropathy in rats. Eur J Pharmacol 2016;5(774):87-94. doi:10.1016/j. ejphar.2016.02.007

15. Zhang XG, Zhang YQ, Cheng QP, et al. The impact of insulin pump therapy to oxidative stress in patients with diabetic nephropathy. Eur J Med Res. 2018;23(1):7. doi:10.1186/s40001-018-0304-2

16. Beckman JA, Goldfine AB, Leopold JA, et al. Ebselen does not improve oxidative stress and vascular function in patients with diabetes: a randomized, crossover trial. Am $J$ Physiol Heart Circ Physiol. 2016;311(6):H1431-H1436. doi:10.1152/ ajpheart.00504.2016

17. Sztanek F, Molnárné Molnár Á, Balogh Z The role of oxidative stress in the development of diabetic neuropathy. Orv Hetil 2016157 (49):1939-1946. doi:10.1556/650.2016.30609

18. Himeno T, Kamiya H, Naruse K, et al. Beneficial effects of exendin-4 on experimental polyneuropathy in diabetic mice. Diabetes. 2011;60 (9):2397-2406. doi:10.2337/db10-1462

19. Shakeel M Recent advances in understanding the role of oxidative stress in diabetic neuropathy. Diabetes Metab Syndr 20159 (4):373-378. doi:10.1016/j.dsx.2014.04.029

20. Ighodaro OM Molecular pathways associated with oxidative stress in diabetes mellitus. Biomed Pharmacother 2018;108:656-662. doi:10.1016/j.biopha.2018.09.058 
21. Terashima T, Kojima H, Chan L Bone marrow expression of poly (ADP-ribose) polymerase underlies diabetic neuropathy via hematopoietic-neuronal cell fusion. FASEB J, 2012;26(1):295-308. doi:10.1096/fj.11-186262

22. Henning RJ, Bourgeois M, Harbison RD Poly(ADP-ribose) polymerase (PARP) and PARP inhibitors: mechanisms of action and role in cardiovascular disorders. Cardiovasc Toxicol 2018 18(6):493-506. doi:10.1007/s12012-018-9462-2

23. Shah AP, Patel CN, Sureja DK, et al. A review on DNA repair inhibition by PARP inhibitors in cancer therapy. Folia Med (Plovdiv) 2018;60(1):39-47. doi:10.1515/folmed-2017-0067
24. Han Y, Wang M, Shen J, et al. Differential efficacy of methylcobalamin and alpha-lipoic acid treatment on symptoms of diabetic peripheral neuropathy. Minerva Endocrinol 2018 43(1):11-18. doi:10.23736/S0391-1977.16.02505-0

25. Agathos E, Tentolouris A, Eleftheriadou I, et al. Effect of $\alpha$-lipoic acid on symptoms and quality of life in patients with painful diabetic neuropathy. J Int Med Res 2018 46(5):1779-1790. doi:10.1177/ 0300060518756540

\section{Publish your work in this journal}

Diabetes, Metabolic Syndrome and Obesity: Targets and Therapy is an international, peer-reviewed open-access journal committed to the rapid publication of the latest laboratory and clinical findings in the fields of diabetes, metabolic syndrome and obesity research. Original research, review, case reports, hypothesis formation, expert opinion and commentaries are all considered for publication. The manuscript management system is completely online and includes a very quick and fair peer-review system, which is all easy to use. Visit http://www.dovepress.com/testimonials.php to read real quotes from published authors. 